\title{
Mitigating staff shortages: Risk of permitting healthcare workers to return to work after coronavirus disease 2019 (COVID-19) exposure
}

\author{
Cherie Faith Lopez Monsalud MPH ${ }^{1}$ (1), Mary Frazier Greene Lind MSN ${ }^{1}$, Carolyn M. Hines BSN ${ }^{1}$, Donna Schora $\mathrm{MT}^{2}$, \\ Jennifer Grant $\mathrm{MD}^{1}$, Erin McElvania $\mathrm{PhD}^{3,4}$ and Kamaljit Singh MD ${ }^{1,3}$ \\ ${ }^{1}$ Department of Infection Prevention and Control, NorthShore University HealthSystem, Evanston, Illinois, ${ }^{2}$ Department of Infectious Diseases Research, \\ NorthShore University HealthSystem, Evanston, Illinois, ${ }^{3}$ Department of Pathology, NorthShore University HealthSystem, Evanston, Illinois and ${ }^{4}$ Department of \\ Pathology, University of Chicago, Pritzker School of Medicine, Chicago, Illinois
}

To the Editor-Healthcare systems are struggling to manage overwhelming patient volumes and an exhausted workforce during the coronavirus disease 2019 (COVID-19) pandemic. Acute healthcare worker (HCW) shortages due to rising rates of COVID-19 infection are further exacerbated by staff exposures requiring furlough from work. In an effort to mitigate staffing shortages, the Centers for Disease Control and Prevention (CDC) recommends forgoing work restrictions after an exposure in favor of universal symptom screening and use of personal protective equipment (PPE). ${ }^{1}$

In November 2020, NorthShore University HealthSystem (NSUHS) faced critical staffing shortages due to the rapid increase of COVID-19 cases in Illinois. Tasked to re-evaluate existing work restriction protocols for COVID-19-exposed HCWs, NSUHS modified its high- and low-risk exposure (LRE) categorization and subsequent quarantine guidance. High-risk exposures (HRE) included (1) HCWs who participated in an aerosol generating procedure of a COVID-19 infected patient without an N95 respirator/PAPR and eye protection and (2) ongoing exposure to a COVID-19-infected household member from whom they cannot self-isolate. ${ }^{2}$ HCWs with HRE are required to self-quarantine and undergo severe acute respiratory coronavirus virus 2 (SARS-CoV-2) RT-PCR (RT-PCR) testing 5 days after their last exposure. ${ }^{3}$ All other exposed HCWs are categorized as LRE and are permitted to continue working, self-monitor for symptoms, and strictly adhere to NSUHS PPE policies. ${ }^{4}$ To assess the safety of allowing HCWs with LRE to continue working, the NSUHS Infection Prevention and Control Department conducted a voluntary surveillance program to determine prevalence of SARS-CoV-2 in asymptomatic HCWs with LRE.

\section{Methods}

Asymptomatic HCWs without history of COVID-19 infection who reported an LRE via electronic questionnaire were asked to undergo RT-PCR testing 5-7 days after exposure. ${ }^{3}$ All participants were prospectively interviewed for previous or current symptoms of COVID-19 infection. RT-PCR positive participants were interviewed again to assess for new symptom development and were asked to complete a 10 -day quarantine. ${ }^{5}$

\footnotetext{
Author for correspondence: Mary Frazier G. Lind, E-mail: Mgreene@Northshore.org Cite this article: Monsalud CFL, et al. (2022). Mitigating staff shortages: Risk of permitting healthcare workers to return to work after coronavirus disease 2019 (COVID-19) exposure. Infection Control \& Hospital Epidemiology, 43: 827-828, https:// doi.org/10.1017/ice.2021.121
}

Participants were provided a nasal swab kit with instructions for specimen self-collection. ${ }^{6}$ The nasal swab consisted of a FLOQswab (Copan Diagnostics, Murrieta, CA) in viral transport medium. RT-PCR testing was performed using the BDMax System (Becton Dickinson, Sparks, MD) and results were available within 12 hours. The goal was to enroll $\sim 100 \mathrm{HCWs}$, but due to the start of COVID-19 vaccination on December 17, 2020, enrollment was stopped early because of the potential for reduced infection and transmission risk among vaccinated HCWs.

\section{Results}

The surveillance program was conducted from November 23 to December 31, 2020, which paralleled a significant increase in staff shortages due to COVID-19 infection and/or exposures (Fig. 1). The number of HCWs undergoing SARS-CoV-2 testing increased from 396 in September to 1,213 in November 2020, with a corresponding increase in positivity rate from $7.8 \%$ to $26.5 \%$.

During the surveillance period, $179 \mathrm{HCW}$ reported an LRE and $73(40.7 \%)$ agreed to provide a self-collected specimen. However, $7 \mathrm{HCWs}$ did not appear for specimen collection, so a total of 66 HCWs with LRE were included in the final analysis. The age range of participants was 22-69 years, with a median of 43.5 years. Most participants were female ( 43 of $66,65.2 \%$ ), worked in a hospital unit $(60$ of $66,91.0 \%)$ and had nonhousehold exposures (37 of $66,56.0 \%)$. The top 5 participant occupations were registered nurse (23 of $66,34.8 \%$ ), physician ( 7 of $66,10.6 \%$ ), respiratory therapist ( 7 of $66,10.6 \%$ ), physician assistant ( 4 of 66 , $6.0 \%$ ), and patient care technician ( 3 of $66,4.5 \%)$. Also, 5 participants $(7.6 \%)$ had a positive RT-PCR. Acute and convalescent SARS-CoV-2 sera were collected in a subgroup of 13 participants for antibody testing (EuroImmun IgG Spike antibody and Roche Nucleocapsid Total antibody). Furthermore, 2 participants were antibody positive in convalescent sera only (both were RT-PCR positive), and 1 was positive in both acute and convalescent sera (RT-PCR negative) due to prior COVID-19 infection in April 2020.

During follow-up interviews, 2 RT-PCR-positive participants remained asymptomatic and 1 developed symptoms after testing. Upon further questioning, 2 positive participants identified COVID-19 compatible symptoms: 1 had a mild cough, anosmia, and malaise 2 weeks before testing that resolved before enrollment. The second developed rhinorrhea and congestion 2 days after enrollment; symptoms that were attributed to an asthma exacerbation. We identified 1 probable nosocomial transmission event in a patient who was negative on admission and asymptomatic and 
Fig. 1. NorthShore University HealthSystem COVID-19 employee testing results March 2020-December 2020.

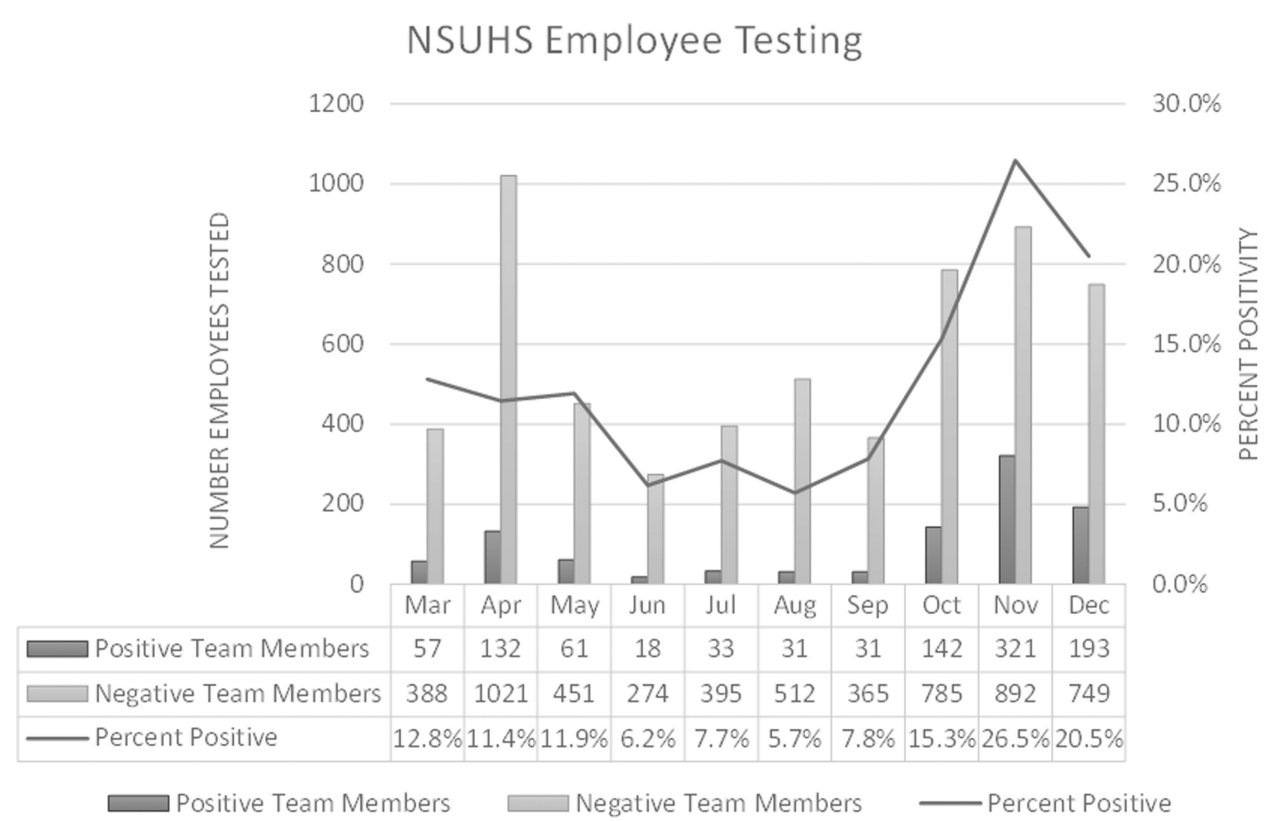

who retested RT-PCR positive on hospital day 25. This patient received care from 2 of the positive HCW participants.

\section{Discussion}

The prevalence of SARS-CoV-2 in HCWs with LRE who continue working is unknown. Our surveillance identified 5 (7.6\%) RTPCR-positive LRE HCWs. Allowing COVID-19-exposed staff to continue working while infected increases the risk of nosocomial SARS-CoV-2 transmission. At least $40 \%$ of COVID-19 transmissions are estimated to be from asymptomatic individuals. ${ }^{7,8}$ The risk of nosocomial transmission is reduced by PPE use but can never be eliminated. We identified 1 probable case of nosocomial COVID-19 infection associated with 2 positive HCWs.

Our study had several limitations. This was a single-center surveillance program. The recruitment period was restricted by the start of the COVID-19 vaccination program on December 17, 2020 , thereby resulting in low participant enrollment. In interviews after testing, we identified 2 participants who had unreported COVID-19 compatible symptoms, which is a limitation of depending on self-reported symptoms. Use of self-collected nasal swabs may provide lower sensitivity, although emerging data suggest that it is likely equivalent to clinician-collected nasopharyngeal swabs. ${ }^{6}$

In conclusion, our surveillance raises concern about allowing LRE HCWs to continue working. Despite universal mask use, asymptomatic HCWs with exposure may pose a significant transmission risk. A follow-up larger study should be conducted in vaccinated and nonvaccinated HCWs to assess risk of allowing HCWs to continue working after exposure. ${ }^{4}$

Acknowledgments. The authors are thankful for support from the Department of Infection Prevention and Control, the Department of Infectious Disease Research, NSUHS Microbiology Laboratories, and the Department of Employee Health.

Financial support. No financial support was provided relevant to this article.
Conflicts of interest. All authors report no conflicts of interest relevant to this article.

\section{References}

1. Strategies to mitigate healthcare personnel staffing shortages. Centers for Disease Control and Prevention website. https://www.cdc.gov/coronavirus/ 2019-ncov/hcp/mitigating-staff-shortages.html. Published December 14, 2020. Accessed December 15, 2020.

2. Interim US guidance for risk assessment and work restrictions for healthcare personnel with potential exposure to COVID-19. Centers for Disease Control and Prevention website. https://www.cdc.gov/coronavirus/2019-ncov/hcp/ guidance-risk-assesment-hcp.html. Published December 14, 2020. Accessed December 15, 2020.

3. Kucirka LM, Lauer SA, Laeyendecker O, Boon D, Lessler J. Variation in false-negative rate of reverse transcriptase polymerase chain reaction-based SARS-CoV-2 tests by time since exposure. Ann Intern Med 2020;173: 262-267.

4. Interim infection prevention and control recommendations for healthcare personnel during the coronavirus disease 2019 (COVID-19) pandemic. Centers for Disease Control and Prevention website. https://www.cdc.gov/ coronavirus/2019-ncov/hcp/infection-control-recommendations.html. Published December 14, 2020. Accessed December 15, 2020.

5. Duration of isolation precautions for adults with COVID-19. Centers for Disease Control and Prevention website. https://www.cdc.gov/coronavirus/ 2019-ncov/hcp/duration-isolation.html. Published October 19, 2020. Accessed December 14, 2020.

6. Therchilsen JH, von Buchwald C, Koch A, et al. Self-collected versus healthcare worker-collected swabs in the diagnosis of severe acute respiratory syndrome coronavirus 2. Diagnostics (Basel) 2020;10(9):678.

7. COVID-19 pandemic planning scenarios. Centers for Disease Control and Prevention website. https://www.cdc.gov/coronavirus/2019-ncov/hcp/ planning-scenarios.html. Published September 10, 2020. Accessed December 15, 2020.

8. Johansson MA, Quandelacy TM, Kada S, et al. SARS-CoV-2 transmission from people without COVID-19 symptoms. JAMA Netw Open 2021; 4(1):e2035057. 